\title{
Manejo de un aneurisma infeccioso
}

\author{
Daniela González H. ${ }^{1}$, Juan Carlos Herrera N. ${ }^{1}$, Sandra Hasbún A. ${ }^{1}$ y Patricio Tepper L. ${ }^{1}$
}

${ }^{1}$ Equipo Cirugía Vascular y Endovascular. Hospital Regional de Talca Dr. César Garavagno Burotto. Servicio de Salud Maule, VII Región,

Recibido el 9 de octubre de 2018 y aceptado para publicación el 18 de octubre de 2018

Correspondencia a: Dra. Daniela González H. danielad9@gmail.com

\section{Treatment of infected aneurysm}

Introduction: Infectious aneurysms are a rare disease and are characterized by infection of the involved vessel wall. The best treatment is definitely not established. Aim: To describe a case of infectious aneurysm with endovascular management. Case report: We present a 75-year-old male patient admitted to a table compatible with acute abdomen and that an infectious aneurysm of the celiac trunk is investigated in the postoperative period, treated with an endoprosthesis and antibiotic management. Discussion: The traditional management of the infectious aneurysm is open surgery that includes the removal or exclusion of the aneurysm and its infected perivascular tissue, but it leads to great morbidity and mortality. Conclusion: The infectious aneurysm can be managed minimally invasively with endovascular technique, with immediate low morbidity, but with long-term results that are still inconclusive.

Key words: infectious aneurysm; endoprosthesis; endovascular technique.

\section{Resumen}

Introducción: Los aneurismas infecciosos constituyen una patología poco frecuente y se caracterizan por presentar infección de la pared del vaso implicado. El mejor tratamiento no está definitivamente establecido. Objetivo: Describir un caso de aneurisma infeccioso con manejo endovascular. Caso clínico: Presentamos un paciente de sexo masculino de 75 años que ingresa por cuadro compatible con abdomen agudo y que en el posoperatorio se pesquisa un aneurisma infeccioso del tronco celíaco, el cual se trata mediante una endoprótesis y manejo antibiótico. Discusión: El manejo tradicional del aneurisma infeccioso es la cirugía abierta que incluye la extirpación o exclusión del aneurisma y de su tejido perivascular infectado, pero conlleva gran morbimortalidad. Conclusión: El aneurisma infeccioso puede ser manejado de forma mínimamente invasiva con técnica endovascular, con baja morbilidad inmediata, pero con resultados a largo plazo aun no concluyentes.

Palabras clave: aneurisma infeccioso; endoprótesis; técnica endovascular.

\section{Introduccion}

Los aneurismas infecciosos constituyen una patología poco frecuente, entre el 1 y $3 \% \%^{1,2}$ de todos los aneurismas, y corresponden a aquellos que presentan infección de la pared del vaso implicado que conlleva a la destrucción de ésta debido a la proliferación bacteriana formando así el aneuris$\mathrm{ma}^{3,4}$. Por su etiopatogenia se encuentran con mayor frecuencia en enfermos inmunodeprimidos o con sepsis coexistente ${ }^{1,5}$.

El mejor tratamiento quirúrgico para ellos no está definido en su totalidad. Son raros, difíciles de tratar y el manejo clásico con debridamiento de tejidos, resección del aneurisma y reemplazo mediante injerto autólogo in situ o revascularización extra anatómica muchas veces no es factible por su localización tóraco-abdominal o para visceral, y requiere revascularización in situ con alta mortalidad ${ }^{1-4}$. Por lo anterior una opción atractiva es la reparación endovascular de la aorta asociada, y según necesidad, procedimientos híbridos.

Presentamos un caso de aneurisma infeccioso de aorta para visceral tratado mediante endoprótesis.

\section{Caso clínico}

Varón de 75 años sin antecedentes conocidos, fumador de más de 20 cigarrillos/día que ingresa 
por cuadro séptico de origen abdominal. Es intervenido quirúrgicamente evidenciando abscesos hepáticos múltiples, asociado a pus subhepático y fibrina interasas, sin hallazgos patológicos en el intestino, por lo que se realiza aseo quirúrgico y toma de biopsia en cuña de lóbulo hepático izquierdo. En el posoperatorio se mantiene con ceftriaxona/metronidazol y se completa estudio etiológico con TC de abdomen y pelvis con contraste (Figura 1), donde se observan múltiples abscesos hepáticos y un pseudoaneurisma de la aorta abdominal a nivel del tronco celiaco, con compromiso de la arteria hepática. Ecocardiograma transesofágico sin vegetaciones. Virus de inmunodeficiencia humana negativo.

Evoluciona con un íleo posoperatorio y alza de parámetros inflamatorios. Se toma nuevo AngioTC a la semana que muestra un rápido crecimiento del aneurisma infeccioso, de $47 \mathrm{~mm}$ a $63 \mathrm{~mm}$ en diámetro anteroposterior (Figura 2).

Teniendo en cuenta la urgencia de la patología vascular, por el rápido crecimiento del aneurisma infeccioso y un dolor abdominal persistente (EVA 9 a 10/10), a pesar de la analgesia, se decide realizar un EVAR (Figura 3). Acceso inguinal derecho y control vascular con elásticos de la arteria femoral común (AFC). Por izquierdo se coloca un introductor de 7 Fr en AFC, realizando posteriormente una angiografía diagnóstica con un pigtail, en proyección oblicua marcando el origen de la arteria mesentérica superior (AMS) que se ubica $19 \mathrm{~cm}$ bajo el término del aneurisma. Heparina 5000 UI ev. Con un catéter guía coronario se cánula el ostium de la AMS. Por el acceso derecho se asciende la endoprótesis de 26 por $80 \mathrm{~mm}$, realizando el despliegue controlado de ésta. Finalmente, angiografía de salida observa exclusión completa del aneurisma, sin endoleak y AMS permeable.

Buena evolución en el posoperatorio, dejando

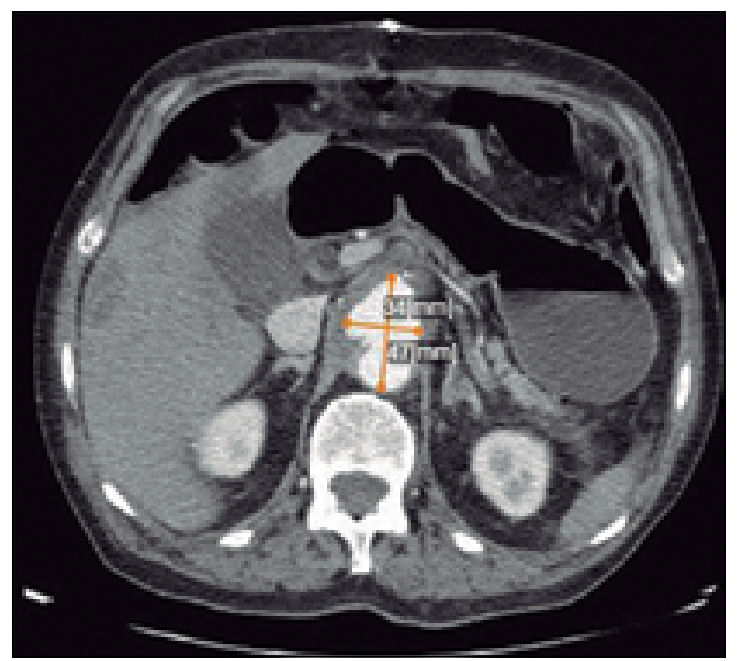

Figura 1. Tomografía computada de abdomen y pelvis donde se observa el aneurisma infeccioso del tronco celiaco que mide 47 por $34 \mathrm{~mm}$ y los abscesos hepáticos.

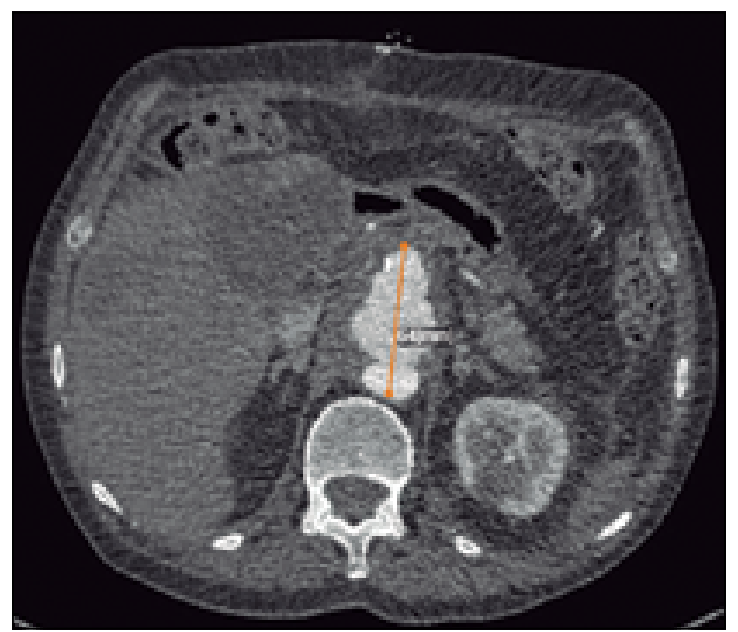

Figura 2. AngioTC de abdomen y pelvis que evidencia la rápida progresión del aneurisma infeccioso, llegando a medir 63 por $40 \mathrm{~mm}$.

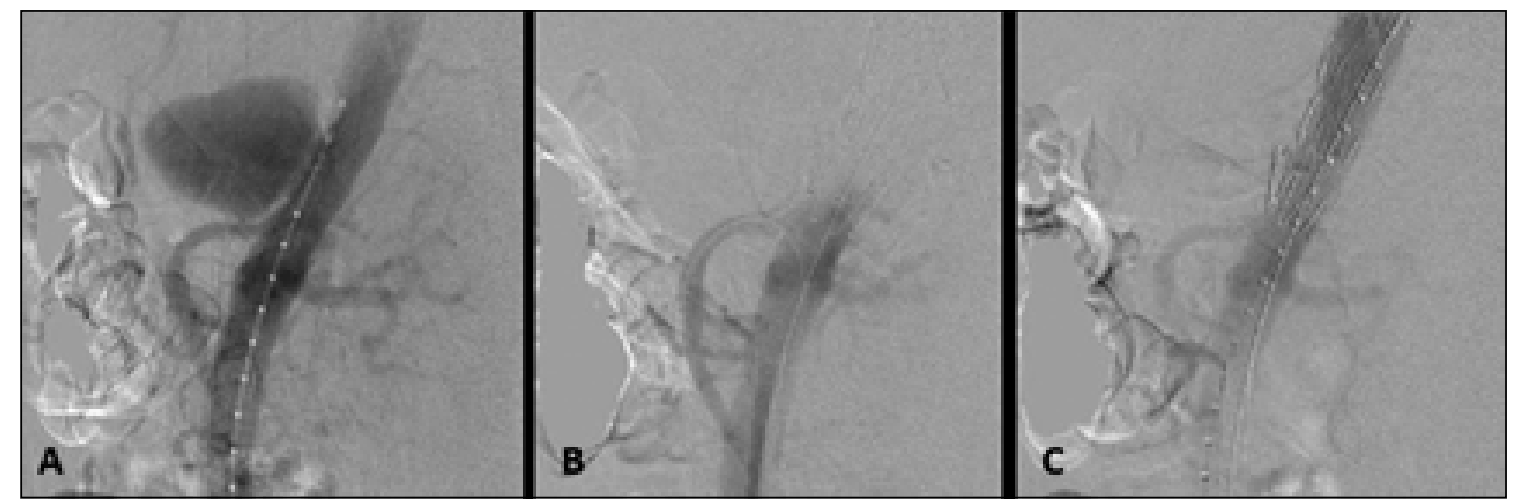

Figura 3. A. Angiografía diagnóstica que muestra el aneurisma infeccioso a nivel del tronco celiaco. B. Protección de la arteria mesentérica superior con catéter coronario. C. Despliegue completo de la endoprótesis observando la exclusión del aneurisma infeccioso y la permeabilidad de la arteria mesentérica superior. 
la unidad de cuidados críticos al día siguiente y reiniciando la alimentación oral. Nunca presentó alteración de las pruebas hepáticas.

Se discute caso con equipo de cirugía digestiva concluyendo que la mejor opción para el manejo de los abscesos hepáticos es mediante drenaje percutáneo, el cual se lleva a cabo sin complicaciones, con la toma de cultivos correspondientes, que resultaron negativos; y la biopsia hepática de la cirugía inicial concluyó abscesos piógenos.

El paciente es dado de alta con tratamiento antibiótico con moxifloxacino $400 \mathrm{mg} /$ día vía oral, recuento leucocitos $5.800 \mathrm{x} \mathrm{mm}^{3}$ y PCR $1,8 \mathrm{mg} / \mathrm{L}$; previamente discutido con infectología y control en el policlínico de cirugía vascular en 30 días.

\section{Discusión}

El término de aneurisma micótico fue introducido por Osler en el siglo XIX y reúne diferentes entidades nosológicas que, actualmente, se denominan en general aneurismas infecciosos. Dependiendo de su etiopatogenia se propone diferenciarlos en cuatro tipos: los aneurismas micóticos procedentes de émbolos sépticos arteriales, la arteritis microbiana con destrucción de la pared y formación de aneurismas, la infección de aneurismas preexistentes y los falsos aneurismas infectados ${ }^{6}$.

La pared arterial normal es resistente a la invasión bacteriana, incluso en los cuadros de bacteremia; sin embargo, en pacientes con inmunodepresión como diabetes mellitus, síndrome de inmunodeficiencia adquirida, desnutrición, cirrosis hepática o en pacientes con defecto luminal arterial (placa o ulceración aterosclerótica, aneurisma preexistente o coartación arterial) la invasión bacteriana en la pared arterial es propensa a través de la vía hematógena ${ }^{5}$. En general, se habla se aneurisma infeccioso, pero siendo rigurosos, se trata en realidad de un pseudoaneurisma.

El diagnóstico se basa en la presentación clínica, laboratorio con elevación de parámetros inflamatorios, cultivos y los hallazgos en la tomografía. Se requiere así la presencia de dos o más de los siguientes hallazgos: sepsis (fiebre, leucocitosis y dolor), hemocultivos positivos, cultivo positivo de la pared del aneurisma y/o hallazgos radiológicos que sugieren infección en la pared ${ }^{6,7}$.

En la cirugía abierta se pueden obtener cultivos positivos del estudio del saco en un 50 a $85 \%$ de los casos, lo que en la modalidad endovascular es aún menor; la obtención de positividad en los hemocultivos es 25 a $79 \%$ lo que delata en estos pacientes una infección más agresiva y asociada a peor pronóstico ${ }^{2,7,8}$.

Dentro de los hallazgos radiológicos para identificar un aneurisma infeccioso están una pared aórtica irregular, signos inflamatorios o masa alrededor del vaso, aneurisma con aire intramural o colección aérea alrededor del vaso, colección líquida perivascular y formación sacular, excéntrica o multilobulada ${ }^{6}$. Si el diagnóstico aun así no es claro deben realizarse escáneres seriados en un corto periodo para evaluar la presencia de crecimiento rápido sugerente de infección ${ }^{5,7}$.

Recientemente, se ha introducido el uso de la tomografía por emisión de positrones (PET- CT) en el diagnóstico y estudio de los aneurismas infecciosos, donde se observa un aumento de la captación aórtica por fluorodesoxiglucosa, correlacionando este hallazgo con una inflamación aórtica e inestabilidad de la pared del aneurisma 5 .

Dada la alta mortalidad sólo con tratamiento médico debido al crecimiento acelerado y por ende mayor probabilidad de ruptura, sin que ésta guarde relación con el diámetro del aneurisma, es que existe consenso de que el enfrentamiento terapéutico debe ser diferente. El manejo quirúrgico tradicional incluye la extirpación o exclusión del aneurisma y de su tejido perivascular infectado; sin embargo, el tipo de cirugía está sujeto a debate ${ }^{1,9,10}$. No existen estudios randomizados que den guías para el tratamiento de los aneurismas infecciosos; su manejo se basa en experiencias clínicas en series de casos. El tratamiento estándar es la antibioterapia más manejo local.

La cirugía convencional presenta elevada morbimortalidad e implica resección del aneurisma, desbridamiento local extenso y revascularización in situ (idealmente con material autólogo) o extra anatómica si es necesaria. Esta última presenta el inconveniente de menor permeabilidad que la revascularización anatómica y si optamos por revascularizar in situ, realizaremos una anastomosis vascular en un lecho infectado, lo que implica riesgo de ruptura por dehiscencia de sutura o falso aneurisma. Aún así, sigue siendo una opción válida para aquellos casos donde el aneurisma infeccioso es relativamente accesible fácilmente en cirugía abierta, como por ejemplo, a nivel iliaco ${ }^{1,2,5,8}$.

La reparación endovascular es menos invasiva, pero no exenta de controversias. Aparte de las limitantes anatómicas (zona de anclaje proximal y distal del endoinjerto), la viabilidad técnica (acceso al vaso) y el tipo de injerto (stents cubiertos versus no cubiertos), el principal factor limitante es la inserción de un cuerpo extraño en un tejido hostil 
infectado, ya que el aneurisma no es resecado ${ }^{8,9,11}$. La presencia del tejido subyacente infectado; a pesar del tratamiento antibiótico, puede mantenerse e infectar la endoprótesis, sumado a que muchas veces nuestros tratamientos antibióticos son empíricos por el bajo porcentaje de ocasiones en que lograremos obtener cultivos ${ }^{3,10,12}$.

Debido a lo anterior, se recomienda el uso de antibióticos previo a la instalación de la endoprótesis y mantenerlos posprocedimiento. Pero no existen recomendaciones definitivas en la literatura en cuanto al tratamiento antibiótico que se debe utilizar ni su duración ${ }^{7,9,12}$.

Por último, cabe mencionar que existe también la opción de procedimientos híbridos, una combinación de la cirugía abierta con la reparación endovascular, representando una solución para tratar los aneurismas que involucran áreas críticas, con difícil acceso por cirugía convencional y además sin necesidad de pinzamiento completo de la aorta ${ }^{13}$.

\section{Conclusión}

El aneurisma infeccioso es una afección potencialmente mortal, con el advenimiento de la cirugía endovascular estamos ofreciendo una solución con mucho menor morbimortalidad a estos pacientes, pero sin tener el conocimiento de la durabilidad de nuestra reparación a pesar de que existen series y casos con resultados alentadores y otros no tanto.

\section{Responsabilidades éticas}

Protección de personas y animales. Los autores declaran que para esta investigación no se han realizado experimentos en seres humanos ni en animales.

Confidencialidad de los datos. Los autores declaran que en este artículo no aparecen datos de pacientes.

Conflictos de interés: no hay.

\section{Bibliografía}

1. Oderich GS, Panneton JM, Bower TC, Cherry KJ Jr, Rowland CM, Noel AA, et al. Infected aortic aneurysms: aggressive presentation, complicated early outcome, but durable results. J Vasc Surg. 2001;34:900-8. DOI:10.1067/ mva.2001.118084.

2. Kyriakides C, Kan Y, Kerle M, Cheshire NJ, Mansfield AO, Wolfe JH. Elevenyear experience with anatomical and extra-anatomical repair of mycotic aortic aneurysms. Eur J Vasc Endovasc Surg. 2004;27:585-9. DOI: 10.1016/j. ejvs.2004.02.024.

3. Sagüés R, Soyo S. Manejo híbrido de aneurisma infeccioso de aorta visceral. Caso clínico. Rev Med Chile 2011;139:1071-4

4. Müller BT, Wegener OR, Grabitz K, Pillny M, Thomas L, Sandmann W. Mycotic aneurysms of the thoracic and abdominal aorta and iliac arteries: experience with anatomic and extra- anatomic repair in 33 cases. J Vasc Surg. 2001;33:106-13. DOI: 10.1067/ mva.2001.110356

5. Young-Wook Kim. Infected Aneurysm: Current Management. Ann Vasc Dis. 2010;3:7-15. DOI: 10.3400/avd. AVDctiia09003.

6. Pérez JL. Mycotic aneurysms: Diagnostic and treatment characteristics. Angiología 2015;68:46-54.

7. Kritpracha B, Premprabha D, Sungsiri J, Tantarattanapong W, Rookkapan S, Juntarapatin P. Endovascular therapy for infected aortic aneurysms. J Vasc Surg. 2011;54:1259-65. DOI: 10.1016/j. jvs.2011.03.301

8. Vallejo N, Picardo NE, Bourke P, Bicknell C, Cheshire NJ, Jenkins MP, et al. The changing management of primary mycotic aortic aneurysms. J Vasc Surg. 2011;54:334-40. DOI: 10.1016/j. jvs.2010.12.066.

9. Kordzadeh A, Watson J, Panayiotopolous YP. Mycotic aneurysm of the superior and inferior mesenteric artery. J Vasc
Surg. 2016;63:1638-46. DOI: 10.1016/j jvs.2016.01.031.

10. Heneghan RE, Singh N, Starnes BW. Successful Emergent Endovascular Repair of a Ruptured Mycotic Thoracic Aortic Aneurysm. Ann Vasc Surg. 2015;29:843.e1-6. DOI: 10.1016/j. avsg.2014.12.035.

11. Abigail Fong A, Navuluri R. Infected Superior Mesenteric Artery Aneurysm. Semin Intervent Radiol. 2016;33:61-4. DOI: $10.1055 / \mathrm{s}-0036-1572358$.

12. Vakhitov D, Suominem V, Korhonen J, Kuorilehto T, Salenius JP. Successful endovascular treatment of mycotic aneurysms of the inferior mesenteric artery and the abdominal aorta. Angiol Sosud Khir. 2016;22:83-8.

13. Marcio F, Da Rocha M, Miranda S, Adriani D, Urgnani F, Riambau VA, et al. Hybrid Procedures for Complex Aortic Pathology: Initial Experience at a Single Center. Rev Esp Cardiol. 2009;62:896-902. DOI: 10.1016/S18855857(09)72654-4. 